\title{
CREENCIAS RELACIONADAS CON LA VIOLENCIA DE PAREJA.
}

\author{
BELIEFS RELATED TO PARTNER VIOLENCE.
}

Ccecarelli Flores Juan ${ }^{1, a}$, Quijandria Angulo Jackeline Estela ${ }^{1, b}$, Quintanilla Bernaola Paul Newman ${ }^{1, b}$, Requejo Torres Jhon Edwin ${ }^{1, b}$

1. Universidad Nacional San Luis Gonzaga de Ica, Perú.

2. Dirección Regional de Salud de Ica, Perú

3. Dirección Regional de Salud de Ayacucho, Perú.

a. Médico Reumatólogo

b. Médico Cirujano

\section{Correspondencia:}

Requejo Torres Jhon Edwin Número de celular: 978060448 Correo Electrónico: dildiwana_@hotmail.com Domicilio: CAsoc. Santa Elvira Mz 1 - lote 7, Ica, Perú.

Contribuciones De Autoría: CFJ, QAJ, QBP y RTJ: Contribuciones en la concepción y diseño del manuscrito, recolección, análisis e interpretación de los datos, redacción y revisión crítica del contenido del manuscrito y aprobación final del artículo.

Conflicto De Intereses: No declarados.

Financiamiento: Autofinanciado.

\section{Como Citar}

Ccecarelli-Flores Juan, QuijandriaAngulo Jackeline Estela, Quintanilla-Bernaola Paul Newman, Requejo-Torres Jhon Edwin, Creencias relacionadas con la violencia de parejaA. Rev méd panacea 2018 ; (2): 56-62

Recibido: 17 - 04 - 2018

Aceptado: 17 - 05 - 2018

Publicado: 15 - 08 - 2018

\section{RESUMEN}

Objetivo: Determinar las creencias relacionadas con la violencia de pareja en los pobladores del Centro Poblado "La Tierra Prometida" (Ica). Materiales y métodos: Se realizó un estudio observacional, descriptivo y transversal en el Centro Poblado "La Tierra Prometida" (Ica), que alberga aproximadamente 1800 familias y 3600 pobladores de 19 a 59 años de edad. La muestra de 361 pobladores se seleccionó mediante muestreo aleatorio multietápico. Resultados: La violencia de pareja fue reportada por 350 (97,0\%) entrevistados, en 345 (95,6\%) de ellos fue de tipo psicológico y en 271 (75,1\%) de tipo físico. Se observó mayor aceptación (de acuerdo o muy de acuerdo) con el enunciado "la mujer no se da cuenta cuando maltrata a su pareja" (75,1\%), "el hombre que maltrata a una mujer tiene problemas psicológicos" $(73,4 \%)$ y "el hombre que se deja maltratar por una mujer tiene problemas psicológicos" (73,2\%). La violencia psicológica se asoció con la actividad laboral y el antecedente de violencia infantil $(p<0,5)$; la violencia física se asoció al grado de instrucción $(p<0,05)$. Conclusiones: Las creencias de la población denotan la presencia de patrones culturales que estigmatizan al hombre frente a este problema y que podrían limitar su reconocimiento y denuncia.

Palabras clave: Palabras clave: Violencia de pareja, adaptación sicológica, conflicto (sicología). (Fuente Decs Bireme)

\section{ABSTRACT}

Objective: To determine the beliefs related to intimate partner violence in the settlers of the "Promised Land" Populated Center (Ica). Materials and methods: An observational, descriptive and cross-sectional study was carried out in the "Promised Land" Town, which houses approximately 1,800 families and 3,600 residents aged 19 to 59 years. The sample of 361 inhabitants was selected by multistage random sampling. Results: Partner violence was reported by 350 (97.0\%) interviewed persons, it was psychological one in 345 (95.6\%) of them and physical type in 271 (75.1\%). Greater acceptance was made according to the statement "the woman does not realize when she mistreats her partner" (75.1\%), "the man who mistreats a woman has psychological problems" $(73,4 \%)$ and "the man who lets himself be mistreated by a woman has psychological problems "(73.2\%). Psychological violence was associated with work activity and a history of childhood violence ( $p$ $<0.5)$; physical violence was associated with the level of education $(p<0.05)$. Conclusions: The beliefs of the population denote the presence of cultural patterns that are stigmatizing the man and that can be limiting its recognition and denunciation.

Keywords: Intimate partner violence, adaptation psychological, conflicto (psychology). (Source: MeSH NLM) 


\section{INTRODUCCIÓN}

La violencia es una conducta intencional, cuyo objetivo es dañar, imponer, vulnerar, reprimir o anular. Posee direccionalidad, ya que siempre va dirigida hacia una persona específica. Puede ser un medio de resolución de conflictos, imponiendo una solución sin utilizar diálogo o negociación; una forma de ejercicio de poder; o una reacción a situaciones irritantes, frustrantes, conflictivas, de peligro o agresión ${ }^{1}$. Es un instrumento de dominio que tiene doble efecto; por un lado, instala la subordinación por medio de la fuerza y, por otro lado, mantiene la subordinación porque la pérdida de recursos y capacidades la facilita, al crear relaciones de dependencia. Así, la violencia se constituye en un instrumento para restringir la libertad y cualquier forma de restricción de la libertad es contraria al desarrollo².

En el mundo, los hombres tienen mayores probabilidades que las mujeres de sufrir violencia en el contexto de conflictos armados y actividades delictivas, mientras que las mujeres tienen mayores probabilidades que los hombres de sufrir violencia y lesiones provocadas por personas cercanas, como esposos y compañeros. Las consecuencias de esa violencia pueden ser duraderas y de amplio alcance, por lo que es una causa importante de lesiones, de enfermedad $y$, en algunos casos, de muerte ${ }^{3}$.

Las mujeres son objeto de violencia en varios ámbitos (hogar, comunidad, instituciones gubernamentales, ámbito productivo y educativo), en distintas etapas de su vida (infancia, adolescencia, edad adulta y vejez) y en diferentes relaciones (de noviazgo y conyugales). Son además objeto de múltiples tipos de violencia (física, sexual, emocional, psicológica, socio-económica, prácticas tradicionales en su contra) ${ }^{7,8}$.

La violencia contra las mujeres en relaciones de parejas es un grave problema de salud pública y un importante obstáculo para el desarrollo socioeconómico de muchos países, principalmente en vías de desarrollo debido a que ocasiona alta carga de morbilidad y mortalidad evitable, requiere un elevado valor presupuestal para atender sus consecuencias en la salud, afecta no solo a las víctimas sino también a la sociedad y genera grandes pérdidas de costo-oportunidad ${ }^{19}$. Es una causa significativa de discapacidad y muerte, entre mujeres de edad reproductiva, tanto en el mundo industrializado como en desarrollo ${ }^{14}$

El estudio publicado el año 2013 por la Organización Mundial de la Salud ${ }^{4}$ reveló que la violencia de pareja es el tipo más común de violencia contra la mujer, debido a que afecta al $30 \%$ de las mujeres en todo el mundo, llegando al 38\% en algunas regiones; la tercera parte de los feminicidios se debe a la violencia conyugal y -en las Américas- el $29,8 \%$ de las mujeres han sido víctimas de violencia física y/o sexual ejercida por parte de su pareja. En América Latina, la violencia doméstica contra la mujer ha ido en aumento en los últimos años. Estamos asistiendo a un problema que hoy en día es catalogado de salud pública ${ }^{20,21}$, de violación de derechos humanos y una barrera para el desarrollo económico ${ }^{20}$. Es un problema muy frecuente que ha llevado a una serie de organismos de la sociedad civil y gobiernos a promover políticas públicas para reducir su frecuencia e impacto sobre la sociedad ${ }^{22,23}$. A pesar que son muchas las campañas que se han realizado en torno al tema, en muchos países latinoamericanos todavía estamos lejos de encontrar una solución ${ }^{20}$.

\section{MATERIALES Y MÉTODOS}

Se realizó un estudio observacional, descriptivo y transversal en el Centro Poblado "La Tierra Prometida" (Ica), que alberga aproximadamente 1800 familias y 3600 pobladores de 19 a 59 años de edad. La muestra se estimó al máximo poder muestral para población conocida (361 pobladores) y mediante muestreo aleatorio multietápico se eligió a los pobladores que participaron en el estudio.

El centro poblado se dividió en dos sectores (norte y sur) usando como delimitación la vía principal que cruza el centro poblado. Cada uno, a su vez, fue dividido en tres subsectores. En estos subsectores se organizaban las viviendas en 250 manzanas (bloques) con un promedio de 13 viviendas por bloque y una mediana de 11. Debido a que el número esperado de pobladores de 19 a 59 años fue 2, se seleccionaron 192 viviendas, adicionando 20 viviendas por posible pérdida de muestra durante la ejecución del estudio. Mediante muestreo aleatorio estratificado se seleccionaron los 16 bloques que albergaban todas las viviendas requeridas en las que se entrevistaron a los 384 pobladores.

Se incluyeron a varones o mujeres de 19 a 59 años de edad, con relación de pareja solteros o casados, independientemente de la convivencia, residentes del Centro Poblado "La Tierra Prometida" que a aceptaron participar en el estudio mediante firma del consentimiento informado.

Se excluyeron a los varones o mujeres de 19 a 59 años de edad, casados o convivientes, separados de la pareja por decisión mutua o judicial, a aquellos que no firmaron el consentimiento informado y a quienes tomaron la decisión de no continuar participando en el estudio.

La recolección de datos se realizará a través de una entrevista realizada por los investigadores y profesionales de enfermería calibrados en la aplicación del instrumento. Cada par de entrevistadores tuvo a cargo una manzana para optimizar el uso del tiempo. La duración aproximada de la entrevista fue de 20 minutos. Al visitar cada vivienda, el entrevistador saludó y se identificó con la persona que respondía o abría la puerta. Luego se le explicó que se estaba realizando un estudio de salud y se le preguntaró ¿cuántas personas de 19 a 59 años de edad habitan en la vivienda? Si la persona tenía 19 a 59 años de edad se le solicitó permiso para hacerle algunas preguntas, caso contrario se le pidió que llame a una persona de ese grupo etario.

Si accedió a continuar, se le preguntó si era residente del Centro Poblado "La Tierra Prometida. Si la respuesta es 
afirmativa se le preguntará si tiene una relación de pareja (esposa o compañero(a)) independientemente de la situación de convivencia.

Se utilizó una ficha ad hoc (ver Anexo $N^{\circ} 1$ ) que constaba de cuatro áreas, correspondientes a: violencia en relaciones de pareja (psicológica o física), creencias relacionadas a violencia en relaciones de pareja, características individuales (edad, sexo, estado civil, grado de instrucción, ocupación, número de parejas previas, tipo de aseguramiento) y antecedente de violencia familiar (en la niñez, contra la madre y contra el padre). Su fiabilidad se constató mediante un estudio piloto con el fin de comprobar si los entrevistados comprenden las preguntas, la terminología y el vocabulario.

Al culminar se verificó que todas las preguntas estén respondidas. No obstante, al finalizar la aplicación de los instrumentos de la investigación se realizó el control de calidad previo a la digitación a fin de identificar posibles errores o vacíos.

Los datos obtenidos, previo control de calidad, fueron ingresados a una base de datos utilizando el software IBM SPSS ${ }^{\circledR}$ (Statistical Package of the Social Science) en su versión 19.0. El procesamiento y análisis de los datos se realizó mediante este programa estadístico.

Se usó la estadística descriptiva a través de la determinación de proporciones de las variables categóricas, así como las medidas de tendencia central para las variables numéricas. El análisis bivariado se realizó usando las pruebas: test $T$ de Student y test de ANOVA para evaluar las diferencias entre dos y tres o más medias, cuando fueron variables numéricas; asimismo, se usó la prueba estadística de Chi2 para asociar proporciones con respecto a las variables categóricas. Se aceptó como relación estadísticamente significativa si el valor $\mathrm{p}$ fue menor de 0,05.

En concordancia con la declaración de Helsinki y al reporte de Belmont, se solicitó el consentimiento informado específico (Anexo 2) y se mantuvo la plena confidencialidad. Así mismo se respetó el anonimato de las y los participantes en el estudio, otorgando un código que defina su identidad, que solo fue conocido por el investigador, además de limitar el acceso a los datos. Para la realización del presente estudio, se comunicó y solicitó la autorización a la Dirección Regional de Salud de Ica.

\section{RESULTADOS}

Tabla 1: Características generales de los entrevistados. Centro Poblado "La Tierra Prometida", 2017.

\section{CARACTERÍSTICAS GENERALES \\ Sexo} n $\%$

Masculino

129

Femenino

232

35,7

\section{Grupo etario}

19 a 29 años

134

64,3

30 a 59 años

\section{Grado de instrucción aprobado}

\begin{tabular}{|c|c|c|}
\hline Ninguno & 3 & 0,8 \\
\hline Primaria & 69 & 19,1 \\
\hline Secundaria & 180 & 49,9 \\
\hline Superior & 102 & 28,3 \\
\hline Sin dato & 7 & 1,9 \\
\hline \multicolumn{3}{|l|}{ Ocupación principal } \\
\hline Desocupado/a, nunca ha trabajado & 8 & 2,2 \\
\hline Desocupado/a, ha trabajado alguna vez & 87 & 24,1 \\
\hline Trabajador/a independiente & 185 & 51,2 \\
\hline Trabajador/a dependiente & 81 & 22,4 \\
\hline \multicolumn{3}{|l|}{ Aseguramiento de salud } \\
\hline Ninguno & 65 & 18 \\
\hline SIS & 197 & 54,6 \\
\hline EsSalud & 89 & 24,7 \\
\hline Otro público & 8 & 2,2 \\
\hline Privado & 2 & 0,6 \\
\hline \multicolumn{3}{|l|}{ Tipo de vínculo } \\
\hline Enamorado/a & 36 & 10 \\
\hline Novio/a & 37 & 10,2 \\
\hline Conviviente & 239 & 66,2 \\
\hline Casado/a & 49 & 13,6 \\
\hline \multicolumn{3}{|l|}{ Antecedente de violencia infantil } \\
\hline Sí & 222 & 61,5 \\
\hline No & 139 & 38,5 \\
\hline
\end{tabular}

Dos de cada tres entrevistados eran de sexo femenino (64,3\%), 62,9\% de 30 a 59 años de edad, 78,2\% con instrucción secundaria o superior, las tres cuartas partes contaba con aseguramiento en salud ( $54,6 \%$ asegurados por el SIS y $24,7 \%$ por la seguridad social), $66,2 \%$ eran convivientes y $61,5 \%$ con antecedente de violencia infantil.

Tabla 2: Violencia de pareja reportada por los entrevistados.

Centro Poblado "La Tierra Prometida", 2017.

\begin{tabular}{lcc} 
PRESENCIA DE VIOLENCIA DE PAREJA & $\mathbf{n}$ & $\%$ \\
\hline Violencia de pareja & 350 & 97 \\
Sí & 11 & 3 \\
No & & \\
Violencia psicológica de pareja & 345 & 95,6 \\
Sí & 16 & 4,4 \\
No & & \\
Violencia física de pareja & 271 & 75,1 \\
Sí & 90 & 24,9 \\
No & \\
\hline
\end{tabular}

La violencia de pareja fue reportada por 350 (97,0\%) de los entrevistados, de los cuales 345 (95,6\%) manifestó que sufrió violencia psicológica de pareja y 271 (75,1\%) violencia física 
Tabla 3: Creencias sobre la violencia de pareja de los entrevistados. Centro Poblado "La Tierra Prometida", 2017

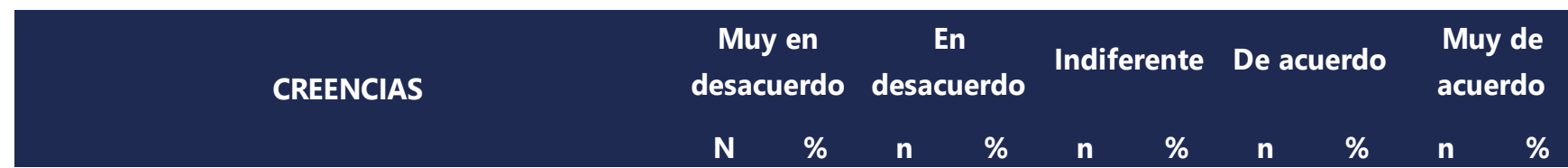

El hombre que maltrata a una mujer tiene problemas psicológicos.

$\begin{array}{llllllllll}10 & 2,8 & 51 & 14,1 & 34 & 9,4 & 225 & 62,3 & 41 & 11,4 \\ 1 & 0,3 & 53 & 14,7 & 36 & 10 & 226 & 62,6 & 45 & 12,5\end{array}$

La mujer no se da cuenta cuando maltrata a su pareja.

El maltrato psicológico no deja secuelas.

$\begin{array}{llllllllll}10 & 2,8 & 54 & 15 & 88 & 24,4 & 163 & 45,2 & 46 & 12,7\end{array}$

La mujer que maltrata a un hombre tiene

problemas psicológicos.

$\begin{array}{llllllllll}47 & 13 & 50 & 13,9 & 48 & 13,3 & 166 & 46 & 50 & 13,9\end{array}$

Sólo el maltrato físico deja secuelas.

El hombre que se deja maltratar por una mujer tiene problemas psicológicos.

Las mujeres que agreden a sus parejas se sienten avergonzadas y culpables por ello.

Los hombres provocan a sus parejas para que pierdan el control.

$\begin{array}{lllllllll}2,8 & 69 & 19,1 & 53 & 14,7 & 187 & 51,8 & 42 & 11,6\end{array}$

El castigo físico es necesario en los niños.

$\begin{array}{llllllllll}57 & 15,8 & 66 & 18,3 & 45 & 12,5 & 149 & 41,3 & 44 & 12,2\end{array}$

Cuando la mujer maltrata a su pareja es porque se preocupa por él.

$\begin{array}{llllllllll}56 & 15,5 & 57 & 15,8 & 58 & 16,1 & 132 & 36,6 & 58 & 16,1\end{array}$

Los hombres que agreden a sus parejas se sienten avergonzados y culpables por ello.

Cuando el hombre maltrata a su pareja es porque se preocupa por ella.

Las mujeres que maltratan a sus parejas o hijos(as) han sido maltratadas por sus padres. La mujer que se deja maltratar por un hombre tiene problemas psicológicos.

Las mujeres provocan a sus parejas para que pierdan el control.

El hombre no se da cuenta cuando maltrata a su pareja. 
La violencia en la pareja ocurre solo cuando existen problemas económicos en la familia.

$\begin{array}{rrrrrrrrrr}38 & 10,5 & 83 & 23 & 36 & 10 & 146 & 40,4 & 58 & 16,1 \\ 9 & 2,5 & 115 & 31,9 & 31 & 8,6 & 141 & 39,1 & 65 & 18\end{array}$

hijos(as) han sido maltratados por sus padres.

La persona que maltrata a su pareja es porque ha consumido alcohol y/o drogas.

17

$\begin{array}{lllllllll}4,7 & 112 & 31 & 44 & 12,2 & 146 & 40,4 & 42 & 11,6\end{array}$

Los celos son la causa de la violencia en la pareja.

$\begin{array}{llllllllll}14 & 3,9 & 91 & 25,2 & 49 & 13,6 & 153 & 42,4 & 54 & 15\end{array}$

Las mujeres sólo pueden agredir a sus hijos.

$$
\begin{array}{llllllllll}
39 & 10,8 & 55 & 15,2 & 32 & 8,9 & 161 & 44,6 & 74 & 20,5
\end{array}
$$

Los hombres sólo pueden ser agredidos cuando son niños o adolescentes.

\begin{tabular}{llllllllll}
64 & 17,7 & 74 & 20,5 & 30 & 8,3 & 129 & 35,7 & 64 & 17,7 \\
\hline
\end{tabular}

Con relación a las creencias se observó mayor aceptación (de acuerdo o muy de acuerdo) con el enunciado "la mujer no se da cuenta cuando maltrata a su pareja" (75,1\%), "el hombre que maltrata a una mujer tiene problemas psicológicos" $(73,4 \%)$ y "el hombre que se deja maltratar por una mujer tiene problemas psicológicos" (73,2\%). Asimismo, aquellas que mostraron mayor desaprobación fueron "los hombres provocan a sus parejas para que pierdan el control" (36,0\%), "el castigo físico es necesario en los niños" (34,1\%) y "cuando la mujer maltrata a su pareja es porque se preocupa por él" (31,3\%) (Tabla 3).

Tabla 4: Características asociadas a la violencia psicológica en relaciones de pareja. Centro Poblado "La Tierra Prometida", 2017.

\begin{tabular}{|c|c|c|c|c|c|c|}
\hline \multirow[t]{3}{*}{ Características } & \multicolumn{4}{|c|}{ Violencia psicológica de pareja } & \multirow{3}{*}{$\begin{array}{c}\text { Chi } \\
\text { cuadrad } \\
\text { o }\end{array}$} & \multirow{3}{*}{$\begin{array}{l}\text { Valo } \\
\text { de } p\end{array}$} \\
\hline & \multicolumn{2}{|c|}{ Sí } & \multicolumn{2}{|c|}{ No } & & \\
\hline & $\mathbf{n}$ & $\%$ & $\mathbf{n}$ & $\%$ & & \\
\hline \multicolumn{7}{|l|}{ Sexo } \\
\hline Masculino & 122 & 94,6 & 7 & 5,4 & 0,468 & 0,494 \\
\hline Femenino & 223 & 96,1 & 9 & 3,9 & & \\
\hline \multicolumn{7}{|l|}{ Grupo etario } \\
\hline 19 a 29 años & 125 & 93,3 & 9 & 6,7 & 2,625 & 0,105 \\
\hline 30 a 59 años & 220 & 96,9 & 7 & 3,1 & & \\
\hline \multicolumn{7}{|l|}{ Grado de instrucción } \\
\hline Primaria o menos & 68 & 94,44 & 4 & 5,56 & 0,225 & 0,636 \\
\hline Secundaria o más & 270 & 95,74 & 12 & 4,26 & & \\
\hline \multicolumn{7}{|l|}{ Actividad laboral } \\
\hline No trabaja & 86 & 90,53 & 9 & 9,47 & 7,737 & 0,005 \\
\hline Sí trabaja & 259 & 97,37 & 7 & 2,63 & & \\
\hline \multicolumn{7}{|l|}{ Aseguramiento en salud } \\
\hline Sí & 285 & 96,28 & 11 & 3,72 & 1,989 & 0,159 \\
\hline No & 60 & 92,31 & 5 & 7,69 & & \\
\hline \multicolumn{7}{|l|}{ Tipo de vínculo de pareja } \\
\hline Enamorado(a)/novio(a) & 68 & 93,15 & 5 & 6,85 & 1,262 & 0,262 \\
\hline Conviviente/Casado(a) & 277 & 96,18 & 11 & 3,82 & & \\
\hline \multicolumn{7}{|c|}{ Antecedente de violencia infantil } \\
\hline Sí & 218 & 98,2 & 4 & 1,8 & 9,418 & 0,002 \\
\hline No & 127 & 91,4 & 12 & 8,6 & & \\
\hline
\end{tabular}


Al analizar las características asociadas a la violencia psicológica de pareja, se observó que 259 (97,37\%) de los que trabajaban y $86(90,53 \%)$ de los que no trabajaban reportaron ser víctimas de violencia psicológica de pareja $(p=0,005)$; de igual forma 218 (98,20\%) de los que tenían antecedente de violencia infantil, y 127 (91,40\%) de aquellos que no reportaron este antecedente, fueron víctimas de violencia psicológica en su relación de pareja $(p=0,002)$. El sexo del entrevistado, el grado de instrucción, el aseguramiento en salud y el tipo de vínculo con la pareja actual no se asociaron significativamente con la violencia psicológica $(p>0,05)$.

Tabla 5: Características asociadas a la violencia física en relaciones de pareja. Centro Poblado "La Tierra Prometida", 2017.

\begin{tabular}{|c|c|c|c|c|c|c|}
\hline \multirow[t]{3}{*}{ Características } & \multicolumn{4}{|c|}{ Violencia física de pareja } & \multirow{3}{*}{$\begin{array}{c}\text { Chi } \\
\text { cuadrad } \\
0\end{array}$} & \multirow{3}{*}{$\begin{array}{l}\text { Valor } \\
\text { de } p\end{array}$} \\
\hline & \multicolumn{2}{|c|}{ Sí } & \multicolumn{2}{|c|}{ No } & & \\
\hline & $\mathbf{n}$ & $\%$ & $\mathbf{n}$ & $\%$ & & \\
\hline \multicolumn{7}{|l|}{ Sexo } \\
\hline Masculino & 102 & 79,1 & 27 & 20,9 & 1,716 & 0,19 \\
\hline Femenino & 169 & 72,8 & 63 & 27,2 & & \\
\hline \multicolumn{7}{|l|}{ Grupo etario } \\
\hline 19 a 29 años & 95 & 70,9 & 39 & 29,1 & 1,984 & 0,159 \\
\hline 30 a 59 años & 176 & 77,5 & 51 & 22,5 & & \\
\hline \multicolumn{7}{|l|}{ Grado de instrucción } \\
\hline Primaria o menos & 67 & 93,06 & 5 & 6,94 & 15,9 & 0 \\
\hline Secundaria o más & 198 & 70,21 & 84 & 29,79 & & \\
\hline \multicolumn{7}{|l|}{ Actividad laboral } \\
\hline No trabaja & 74 & 77,89 & 21 & 22,11 & 0,55 & 0,458 \\
\hline Sí trabaja & 197 & 74,06 & 69 & 25,94 & & \\
\hline \multicolumn{7}{|l|}{ Aseguramiento en salud } \\
\hline Sí & 48 & 73,85 & 17 & 26,15 & 0,154 & 0,695 \\
\hline No & 233 & 76,14 & 73 & 23,86 & & \\
\hline \multicolumn{7}{|l|}{ Tipo de vínculo de pareja } \\
\hline Enamorado(a)/novio(a) & 54 & 73,97 & 19 & 26,03 & 0,059 & 0,808 \\
\hline Conviviente/Casado(a) & 217 & 75,35 & 71 & 24,65 & & \\
\hline \multicolumn{7}{|c|}{ Antecedente de violencia infantil } \\
\hline Sí & 174 & 78,4 & 48 & 21,6 & 3,373 & 0,066 \\
\hline No & 97 & 69,8 & 42 & 30,2 & & \\
\hline
\end{tabular}

La violencia física se observó en 67 (93,06\%) de los entrevistaos con instrucción primaria o menos y en 198 (70,21\%) de los que tenían instrucción secundaria o superior hubo violencia física de pareja $(p=0,000)$. El sexo masculino, el grado de instrucción, la actividad laboral, el aseguramiento en salud, el tipo de vínculo de pareja y el antecedente de violencia infantil no se asociaron con el reporte de violencia física en la actual relación de pareja $(p>0,05)$. 


\section{REFERENCIAS BIBLIOGRÁFICAS}

1. Burton JP, Hoobler JM. Aggressive reactions to abusive supervision: The role of interactional justice and narcissism. Scandinavian Journal of Psychology. 2011; 52:389-398.

2. Vara-Horna AA, et al. Modelo de gestión para prevenir la violencia contra las mujeres: Una propuesta integral para involucrar a las empresas en la prevención de la violencia contra las mujeres en relaciones de pareja. Lima: ComVoMujer \& Universidad de San Martín de Porres. 2015. pp 168.

3. Guedes A, García-Moreno C, Bott S. Violencia contra las mujeres en Latinoamérica y el Caribe. Foreign Affairs Latinoamérica. 2014; 14(1):41-48.

4. García-Moreno C, Pallitto C, Devries K, Stockl H, Watts $\mathrm{Ch}$, Abrahams N. Estimaciones mundiales y regionales de la violencia contra la mujer: prevalencia y efectos de la violencia conyugal y de la violencia sexual no conyugal en la salud. Resumen de orientación. Ginebra: Organización Mundial de la Salud. 2013.

5. Godoy RM, Ramos AM, Bagueira AE, Skubs T, Núñez R, Yoshikawa E. Violencia doméstica contra la mujer en la visión del agente comunitario de salud. Rev. Latino-Am. Enfermagem. 2009; 17(6):1-13.

6. Miranda MP, de Paula CS, Bordin IA. Violência conjugal física contra a mulher na vida: prevalência e impacto imediato na saúde, trabalho e família. Rev Panam Salud Publica. 2010;27(4):300-8.

7. Butchart A, García-Moreno C, Mikton C. Prevención de la violencia sexual y violencia infligida por la pareja contra las mujeres: qué hacer y cómo obtener evidencias. Washington, DC: Organización Panamericana de la Salud. 2011.

8. and Clinical Psychopharmacology. 2009; 17:154-164.

9. Organización Mundial de la Salud. Preventing intimate partner and sexual violence against women: Taking action and generating evidence. Ginebra, 2010.
10. Watkins L, Jaffe A, Hoffman L, Gratz K, Messman-Moore T, DiLillo D. The longitudinal impact of intimate partner aggression and relationship status on women's physical health and depression symptoms. Journal of Family Psychology. 2014; 28(5):655-665.

11. Bott B, Guedes A, Goodwin M, Adams J. Violencia contra las mujeres en América Latina y el Caribe: análisis comparativo de datos poblacionales de 12 países. Washington, DC: Organización Panamericana de la Salud. 2014.

12. Adelman M, Hea-Kil S. Dating conflicts: rethinking dating violence and youth conflict. Violence Against Women. 2007;13:1296-318.

13. Pontón J. Feminicidio en el Ecuador: realidad latente e ignorada. Ciudad Segura. 2009;31:4-9.

14. Fannin $J L$, Williams RM. La neurociencia de vanguardia revela correlaciones importantes entre las creencias, el estado global del cerebro, y la psicoterapia. Programa Lince. Madrid, España. 2012.

15. López-Angulo $L, \quad F u n d o r a-Q u i n t e r o \quad Y$, Valladares-González A, Ramos-Rangel $Y$, Blanco-Fleites Y. Prevalencia de la violencia física y psicológica en relaciones de pareja heterosexuales. Revista Finlay. 2015; 5(3):161-168.

16. INEI. El $68,2 \%$ de las mujeres fueron víctimas de violencia psicológica, física y/o sexual, alguna vez por el esposo o compañero. 2017. Disponible en: https://www.inei.gob.pe/prensa/noticias/el-682-de-I as-mujeres-fueron-victimas-de-violencia-psicologic a-fisica-yo-sexual-alguna-vez-por-el-esposo-o-com panero-9772/

17. Instituto Nacional de Estadística e Informática. Evolución de la pobreza monetaria 2009-2015. Informe Técnico (internet). Disponible en: https://www.inei.gob.pe/media/MenuRecursivo/pub licaciones_digitales/Est/Lib1347/libro.pdf

\section{Las ediciones anteriores de revista médica PANACEA están disponibles en:}

\section{www.revpanacea.unica.edu.pe}

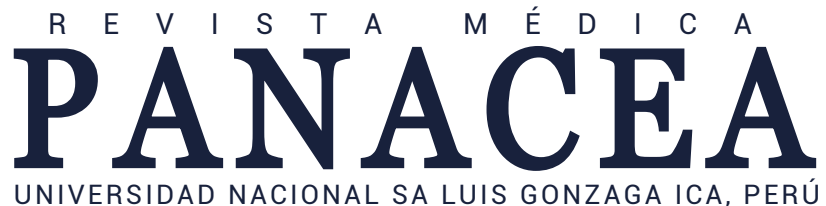

